\title{
Structural Health Monitoring of Different Geometry Structures With Optical Fiber Sensors
}

\author{
Paulo ANTUNES ${ }^{1,2^{*}}$, Hugo RODRIGUES ${ }^{3}$, Rui TRAVANCA ${ }^{3}$, \\ Licínio FERREIRA ${ }^{2}$, Humberto VARUM ${ }^{3}$, and Paulo ANDRÉ ${ }^{1,2}$ \\ ${ }^{1}$ Instituto de Telecomunicações, Campus de Santiago, 3810-193 Aveiro, Portugal \\ ${ }^{2}$ Departamento de Física, Universidade de Aveiro, Campus de Santiago, 3810-193 Aveiro, Portugal \\ ${ }^{3}$ Departamento de Engenharia Civil, Universidade de Aveiro, Campus de Santiago, 3810-193 Aveiro, Portugal \\ *Corresponding author: Paulo ANTUNES_E-mail: pantunes@av.it.pt
}

\begin{abstract}
In this paper, we describe the structural health monitoring of several structures, with different geometry, materials and behaviors, using optical fiber sensors. Those studies aimed to demonstrate the feasibility of such technologies in structural health monitoring, with all the advantages inherent to the optical fiber technology.
\end{abstract}

Keywords: Structural health monitoring, optical fiber, optical sensors

Citation: Paulo ANTUNES, Hugo RODRIGUES, Rui TRAVANCA, Licínio FERREIRA, Humberto VARUM, and Paulo ANDRÉ, "Structural Health Monitoring of Different Geometry Structures With Optical Fiber Sensors," Photonic Sensors, DOI: 10.1007/s13320-012-0094-z.

\section{Introduction}

Buildings, bridges, dams, towers and tunnels, among many other constructions, are very complex engineering structures, but crucial for the prosperity of a modern society. However, those are many times exposed to severe environmental and/or man-made aggressions and harsh loads, resulting in structural degradation. Ensuring the safety, durability and optimizing maintenance costs is a priority. Engineers had been exploiting the advantages of the sensing technologies to identify structural damage and adjust maintenance strategies. Structural health monitoring (SHM) tracks the health status of a structure, merging sensors and monitoring schemes with damage detection systems, to ensure the safety and longevity $[1,2]$. The aim of the SHM is to observe in-situ the structural performance throughout a predetermined short time window or during the structure life time, under diverse loading conditions. This allows detecting the structural or the material properties deterioration and evaluating the safety of the structure. Founded on the monitoring results, an intervention strategy is established in case of need. SHM uses cutting-edge sensors to measure the loading conditions, material and structural properties through structural response parameters (displacement, acceleration, etc.). Anyway, precautionary evaluation can identify problems prematurely and point out recommendations for the solution [3-8].

Among several sensing technologies, optical fiber sensors establish themselves among the most promising for SHM systems. The use of such devices in SHM has increased expressively, due to

Received: 17 August 2012 / Revised version: 3 September 2012

(C) The Author(s) 2012. This article is published with open access at Springerlink.com 
their advantages over the conventional electronic solutions. Compact size, high sensibility, good linearity, immunity to electromagnetic fields, electric isolation, resistance to hash environments and the possibility to be embedded into structures are some of the benefits. For large structures, the multiplexing capacity of fiber Bragg gratings (FBGs) and their high signal-to-noise ratio due to the wavelength encoded information, make them especially attractive [9-11]. With different characteristics from the standard silica fiber $[2,12]$, the polymeric optical fiber (POF), is now being used in innovative developments [13]. The POF allows realizing low-cost sensing due to the lower cost of less complex interrogation systems, the splicing and polishing equipment, the simpler connectors due to its high core diameter and can use less expensive standard visible optical sources and photodetectors [14].

This paper is organized as follows: after an introduction, mentioning the aim of SHM and advantages of optical fiber sensors, the developed and calibrated sensors are presented in Section 2. The description of the studied structures and experimental results is presented in Section 3. Finally, the main conclusions are drawn.

\section{Sensors description}

Within this research line, several optical sensors were developed, mainly based on the FBG technology and POF. Static optical FBG sensors were developed for measuring deformation on an adobe wall during a destructive laboratory test. In a steel footbridge test and in an adobe wall test, a uniaxial FBG accelerometer was used for estimating the structure eigenfrequencies from acceleration data recorded in a short time period. On an elevated water reservoir and on a radio communication tower, a biaxial FBG based accelerometer was used. For monitoring the concrete curing process, a POF based sensor was developed and tested.
An FBG is a passive optical fiber device which is based on the modulation of the fiber core refractive index. When an FBG is illuminated with a spectral broad optical source, it will successively reflect a spectral band of the incident radiation, being the remaining signal transmitted. The reflected signal is a narrow spectral band centered at the Bragg wavelength, $\lambda_{B}$, which is given by the first order Bragg condition [11]:

$$
\lambda_{B}=2 n_{\text {eff }} \Lambda
$$

where $n_{\text {eff }}$ is the refractive index of the optical fiber, and $\Lambda$ is the periodicity of refractive index modulation. By starting on the Bragg condition, a temperature and strain dependence of $\lambda_{B}$ can be expected due to the dependence of $n_{\text {eff }}$ and $\Lambda$ on the temperature and mechanical deformation. This way, stretching or exposing the fiber to temperature variations induces a shift in the reflection spectrum, proportional to the surrounding variation.

The FBG displacement sensors used in the adobe structure test consisted of a fiber Bragg grating attached to two supports, one rigidly fixed and the other movable. The movable support allows the pre-tension application in the fiber for the measurement of expansion and contraction during the structure loading [15].

The uniaxial optical FBG accelerometer used on the footbridge and adobe wall monitoring consisted of a brass inertial mass, supported by an L-shaped cantilever element, which was connected to the sensor support base by a thin steel leaf spring and a Bragg grating element. Additional details on the accelerometer design can be found in [9]. If the accelerometer is exposed to external acceleration, it will cause the inertial mass movement in the sensitive axis, consequently imposing a contraction or expansion of the optical fiber with the FBG [16]. The sensor sensitivity depends on the cantilever dimensions, the inertial mass, the thickness of the leaf spring and the optical fiber Young modulus [2, 9]. The low-cost interrogation system used included 
a band pass optical filter with a linear spectral transfer function in the wavelength change range of the FBG. When the reflected wavelength signal from the FBG changes, it induces variations proportional to the wavelength shift in the optical power transmitted through the optical fiber (related to the convolution of the filter transfer function and the FBG reflection spectrum).

The biaxial optical accelerometer is also based on the FBG technology. It makes use of four multiplexed FBGs into the same optical fiber placed in opposite positions of the inertial mass. The system encompasses two machined aluminum blocks, connected to a rigid base by four bolts and by the four fiber optic Bragg gratings at the top. The fibers are linked between the two blocks with epoxy. The fibers are pre-tensioned before bonding [17]. The inertial mass is composed of the top of the central cylindrical aluminum element, presenting a cylindrical shape at its bottom with 4-mm diameter to allow the inertial mass movement in two independent directions. This movement causes a stretching/compression in the optical fibers and consequently a measurable shift in the FBGs reflected wavelength, related to the external acceleration at the accelerometer base. The external acceleration was measured by the difference of wavelengths shift reflected by two gratings in each direction. In this way, the sensitivity increased by a factor of 2, compared to an FBG based accelerometer using only one FBG per direction. The Bragg gratings were written into the same optical fiber, so they had the same temperature sensitivity, resulting in a wavelength shift similar in both FBGs per direction when subjected to environmental temperature variations. Consequently, the accelerometer is insensitive to temperature variations.

The monitoring of the concrete curing process is highly important, namely in large civil engineering infrastructures, like bridges, dams and tunnels, allowing to control, predict and optimize the formwork placement and removal operation. The rigorous control of the curing process can also prevent accidents and optimize costs [18]. The sensing system used for this purpose was composed of an optical signal from a light emitting diode, injected in a large diameter $(1 \mathrm{~mm})$ POF, with a length of $1 \mathrm{~m}$, unjacketed and embedded in the concrete to be monitored. The method was based on the determination of the water content, based on the scattering of the optical signal in grooves imposed on the fiber, which consequently changed the transmitted optical power through the POF to the receiver, as a result of an increase in the optical loss due to the scattering of the signal [18]. At the initial stage, the grooves were partially filled with the fresh concrete mixture, permitting the signal confinement inside the fiber. As the water percentage reduces during the curing of the concrete, the scattering of the signal increases, and consequently the intensity of the transmitted signal decreases. When the detected power tended asymptotically to a fixed value, the cure process was considered stabilized.

\section{Studied structures and results}

In this section, we present the monitoring of a destructive test on an adobe wall, the dynamic monitoring of a footbridge, an elevated water reservoir and a radio-communications tower, and also the concrete curing process with a POF sensing system is presented.

\subsection{Adobe wall}

In order to simulate the in-plane response of a structural wall connected to perpendicular walls at its edges, an adobe wall specimen with an I-shape geometry was constructed and tested. It was built with adobe blocks obtained from a demolition at Paço, Aveiro, with average dimensions of $(29 \times 45 \times 12) \mathrm{cm}^{3}$. The wall specimen model presented a height of $3.07 \mathrm{~m}$, a length of $3.50 \mathrm{~m}$ and a thickness of $0.33 \mathrm{~m}$ and was the representative of 
the typical adobe constructions in the region. An additional vertical load of 2 tons was placed over the wall, simulating dead-loads, such as roofs and the corresponding live-loads $[15,19]$.

A network of thirteen FBG relative displacement sensors was implemented and effectively interrogated, allowing monitoring the full-scale abode wall destructive test. In order to measure the deformations caused by the horizontal cyclic forces imposed by a hydraulic jack fixed on the west face of the wall, along the direction west-to-east, several relative FBG displacement sensors were used (4 vertical sensors at the wall base, 3 vertical sensors at one wall-flange to measure the flexural response of the wall base, and 5 sensors at the center of the north face to measure locally the distortions, also an FBG displacement sensor was used to measure the horizontal displacement of the east side of the wall). Figure 1 shows a global schematic view of the wall with the sensors positioning. For the dynamic parameters measurement, the uniaxial accelerometer was used, simultaneously with a seismograph and an electronic accelerometer, for the validation of results.

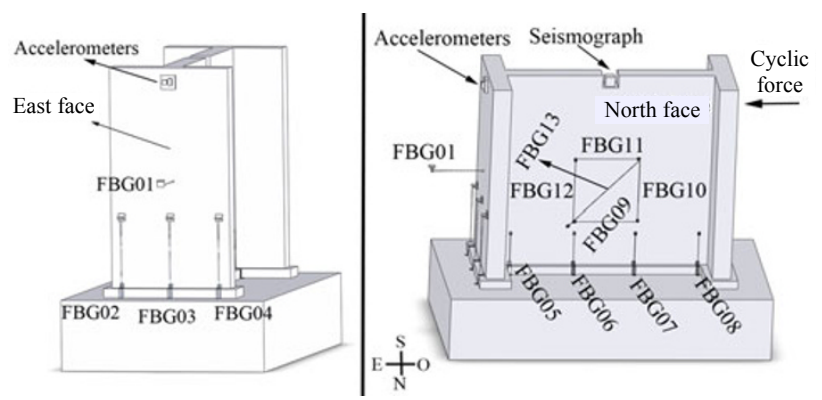

Fig. 1 Schematics with the position of the sensors.

As an example, Fig. 2 shows the evolution of the imposed horizontal cyclic force and a static sensor response. Figure 3 shows the evolution of the response measured at the displacement sensor FBG08 and the horizontal force applied, before the formation of the first crack. From the data presented in Fig. 3, it is possible to observe the good agreement between the deformation measured at the sensor and the applied cyclic force until the first crack in the wall occurs.

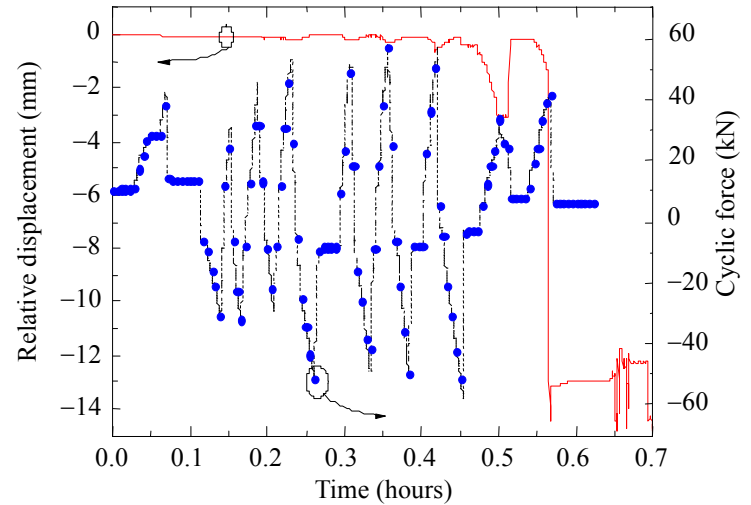

Fig. 2 Imposed cyclic force and FBG01 static sensor response: the line-dot represents the cyclic force, and the line represents the sensor response.

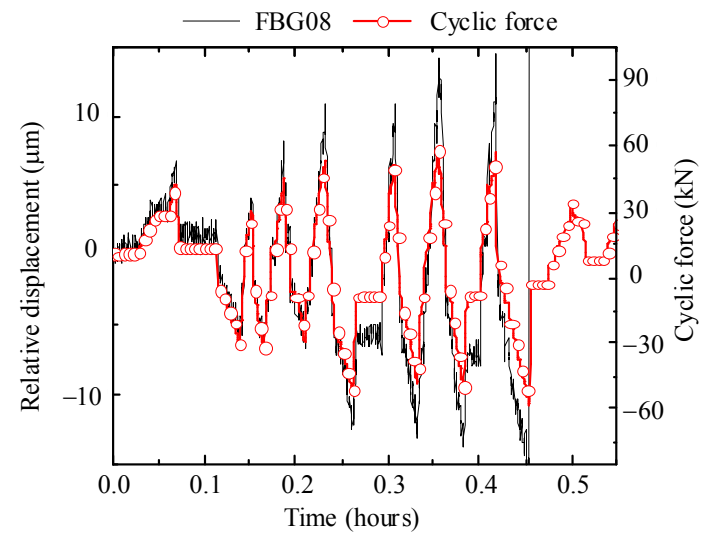

Fig. 3 Displacement sensor FBG08 response and horizontal force applied, before formation of the first crack.

Applying dynamic impulses on the wall in the west-east direction, the response of the structure in terms of the acceleration over time was acquired, using the three acceleration sensors mentioned earlier. This method was repeated for different damage states, before and during the destructive cyclic test. Table 1 displays the estimated first natural frequency value of the wall during the test.

Data in Table 1 shows a decrease in the first natural frequency of the structure, in its longitudinal direction, with the evolution of damage during the destructive test. This result was expected, since the loss of stiffness associated with damage entailed a 
reduction in the natural frequency of any type of structure. Regarding the behavior of the wall, a $34.07 \%$ decrease of the value of the first natural frequency between the baseline (no damage) and the end of the cyclic test (damaged structure) was observed. Furthermore, when cracks arised in the wall, it was noticed in the data recorded by static measurement. The results showed that optical sensors networks could be used as a tool in the support for the SHM of complex systems.

Table 1 Natural frequency evolution along the test.

\begin{tabular}{c|c|c|c}
\hline & \multicolumn{3}{|c}{ Frequency (Hz) } \\
\hline Test conditions & FBG sensor & Seismograph & Relative difference (\%) \\
\hline Before test & 23.08 & 22.89 & 0.83 \\
\hline After 1st cycle & 22.41 & 22.06 & 1.59 \\
\hline After 3st cycle & 21.88 & 21.55 & 1.53 \\
\hline After 1st large crack & 17.94 & 17.79 & 0.84 \\
\hline After 2st large crack & 15.41 & 15.07 & 2.26 \\
\hline End of the test & 15.69 & 15.38 & 2.02 \\
\hline
\end{tabular}

\subsection{Steel footbridge}

The structure studied and presented in this section is a pedestrian bridge located in the campus of the University of Aveiro. This structure makes the connection between the cores of the University Campus of Santiago and the Crasto Campus, over the Esteiro of São Pedro. The deck of the bridge is formed by a tubular steel truss with a constant height of $4 \mathrm{~m}$ and a width of $3 \mathrm{~m}$, with elements made of " $H$ " profile sections. The deck slab is made of reinforced concrete with the $15-\mathrm{cm}$ thickness and 3-m width, supported on two longitudinal beams, with I-section. The eight supporting pillars are spaced $36 \mathrm{~m}$, and the total length of the structure is $324 \mathrm{~m}$ [9].

For obtaining the natural frequencies associated with the vertical modes of the footbridge, the uniaxial optical fiber Bragg grating based accelerometer was used with a commercial interrogation system from Fibersensing (model
FS4200) at a sampling rate of $200 \mathrm{~Hz}$. As a reference, a uniaxial electronic accelerometer from Crossbow (CLX02LF1Z) was used. The natural frequencies of the structure were obtained by peak-picking in the frequency spectrum, obtained by the Fourier transform from the acceleration data over time recorded with both sensors. Figure 4 shows the frequency spectrum resulting from the acceleration data recorded.

Table 2 summarizes the estimated values and the relative difference between the values estimated from the FBG sensor and the electronic one.

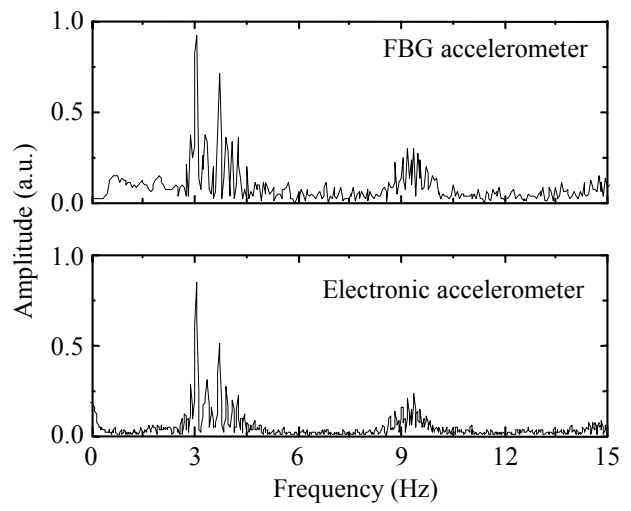

Fig. 4 Frequency spectrum obtained by the Fourier transform from the acceleration data over time.

Table 2 Natural frequencies of the footbridge.

\begin{tabular}{c|c|c|c}
\hline & \multicolumn{3}{|c}{ Frequency (Hz) } \\
\hline Mode & FBG sensor & Electronic sensor & $\begin{array}{c}\text { Relative } \\
\text { difference (\%) }\end{array}$ \\
\hline 1 & 3.076 & 3.052 & 0.799 \\
\hline 2 & 3.320 & 3.345 & 0.729 \\
\hline 3 & 3.711 & 3.711 & $<0.001$ \\
\hline 4 & 3.906 & 3.931 & 0.621 \\
\hline 5 & 4.102 & 4.102 & $<0.001$ \\
\hline 6 & 4.248 & 4.248 & $<0.001$ \\
\hline
\end{tabular}

From the data presented in Table2, a maximum difference of $0.8 \%$ was obtained in estimating the natural frequencies of the structure with the acceleration data measured with the optical accelerometer, relatively to the ones estimated from the measurements obtained with the electronic 
accelerometer.

\subsection{Concrete elevated reservoir}

To estimate the natural frequency of the elevated water reservoir, we used the biaxial FBG accelerometer described above. The reinforced concrete structure is located in the campus of the University of Aveiro. It was constituted by a 30.9-m-height concrete blade with a width of $4 \mathrm{~m}$ and a thickness of $0.3 \mathrm{~m}$, a concrete tubular section element with the same height as the blade, an internal diameter of $1.2 \mathrm{~m}$ and a thickness of $0.6 \mathrm{~m}$. On the top of these vertical structural elements, there is the water reservoir with hollow parallelepiped geometry. At each third of the height, a connection between the blade and tubular section exists, made of a stainless steel bar with a hollow section with an external diameter of $150 \mathrm{~mm}$ (thickness: $5 \mathrm{~mm}$ ). The structure presents a total height of $35.25 \mathrm{~m}$. The FBG accelerometer was fixed behind the metallic access stairway, on the top of the structure with four stainless steel bolts connecting the sensor and a metallic supporter made for this propose [20].

The response to the wind excitation in terms of the acceleration was registered, and the natural frequencies in the transversal and longitudinal directions were estimated from the frequencies spectra, obtained by fast Fourier transform (FFT) of the acceleration data over time. With this test, only with the environmental excitation, it was possible to estimate the structure natural frequencies. This test demonstrates that with the used sensor it is possible to remotely test and monitor this type of structures, without the need for other type of external load. The acceleration, by measuring the wavelength shift of the FBGs, was acquired using a commercial interrogation monitor model I-MON E, from Ibsen Photonics, at a sampling rate of $950 \mathrm{~Hz}$. The frequency spectra obtained are showed in Fig. 5.

The first natural frequency in the transversal direction was estimated to be $0.903 \mathrm{~Hz}$, and in the longitudinal direction the value of $0.805 \mathrm{~Hz}$ was obtained. The values estimated were consistent with the values obtained with external excitations [20]. Those results showed that with these sensors a good estimation of the structure natural frequencies could be reached in remote monitoring schemes, without the external load at the site.

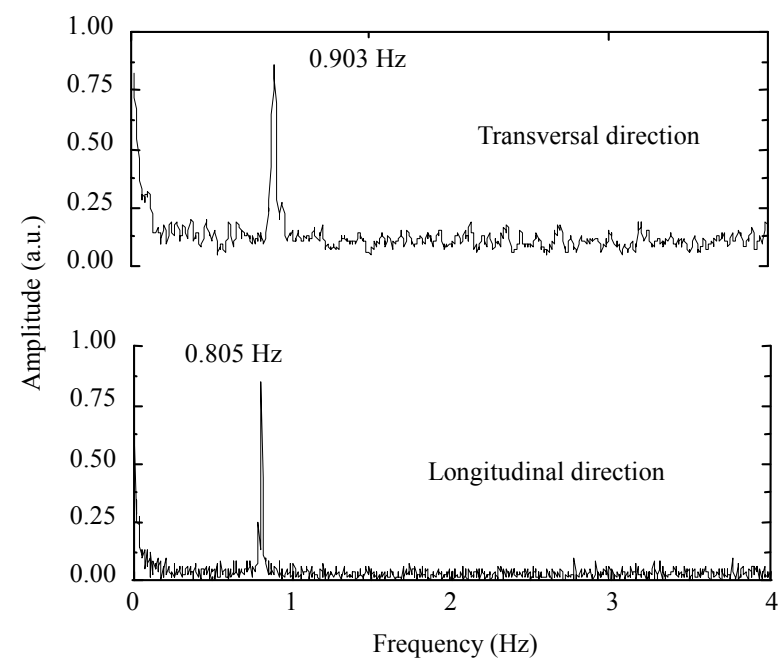

Fig. 5 Frequency spectrum obtained from the acceleration data over time.

\subsection{Radio communication tower}

The towers category varies across countries according to the use and actions to be considered in their design and application. In this work, a 50-m-height monopole was studied. This monopole is constituted by five different steel modules with a tubular cross section joined by bolted connections, fixed at its base to a reinforced concrete foundation slab with the dimensions of $(4 \times 4) \mathrm{m}^{2}$ and a height of $2.25 \mathrm{~m}$.

The FBG biaxial accelerometer adopted in the measurement was fixed on the top of the tower, near the antennas, with a customized support, and external excitation mechanical impulses were applied in the mid-section of the tower. The acceleration data were measured in two perpendicular directions parallel to the ground. The acceleration data were acquired using a commercial 
interrogation monitor model I-MON E, from Ibsen Photonics, at a sampling rate of $950 \mathrm{~Hz}$. From the acceleration data over time by FFT, and the eigenfrequencies of a structure were estimated for both sensitive directions. The frequencies spectra, for both directions, are presented in Fig. 6 .

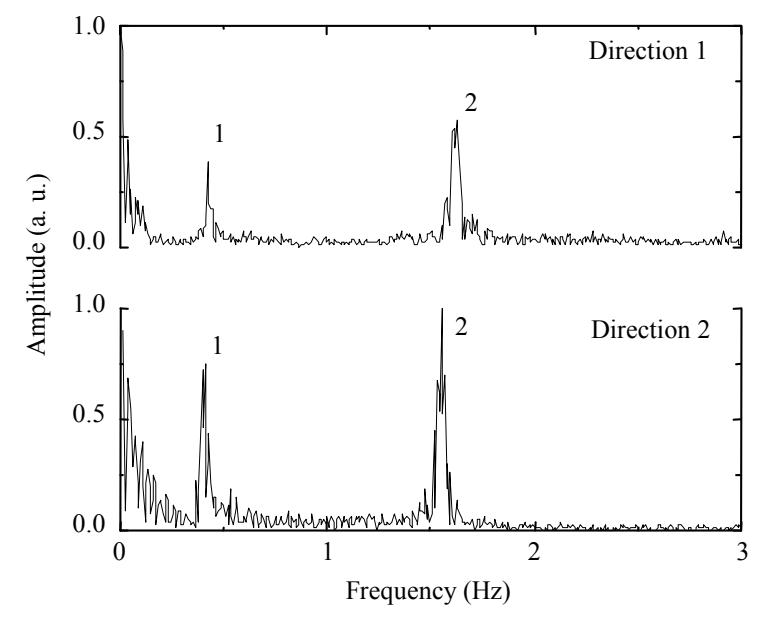

Fig. 6 Frequency spectrum obtained from the acceleration data over time, for both sensitive directions.

From the data in Fig. 6, the estimated natural frequencies obtained are presented in Table 3, for the first three modes.

Table 3 Natural frequencies of the tower.

\begin{tabular}{c|c|c}
\hline \multirow{2}{*}{ Peak number } & \multicolumn{2}{|c}{ Frequency $(\mathrm{Hz})$} \\
\cline { 2 - 3 } & Direction 1 & Direction 2 \\
\hline 1 & 0.429 & 0.414 \\
\hline 2 & 1.628 & 1.555 \\
\hline
\end{tabular}

The estimated values for natural frequencies obtained with the FBG biaxial accelerometer were within the values expected for this type of structures and were also consistent with the results obtained with the numerical modeling [21].

\subsection{Concrete curing monitoring}

The used optical sensor consisted of a 1-m-length POF with 6 grooves equally spaced. The sensor system for the test comprised a light emitting diode (LED), model IFE93 from Industrial Fiber Optics, and the optical receiver was a photodiode, model IFD91 from Industrial Fiber Optics, followed by an electrical transimpedance and amplification stage. The concrete composition was: $8190 \mathrm{ml}$ of water, $16.90 \mathrm{~kg}$ of cement class $32.5,29.90 \mathrm{~kg}$ of gravel (type 12/25), $16.90 \mathrm{~kg}$ of gravel (type 8/12) and $42.25 \mathrm{~kg}$ of sand. The concrete mixture was placed in a cubic mold sizing $15 \mathrm{~cm} \times 15 \mathrm{~cm} \times 15 \mathrm{~cm}$. Figure 7 displays the optical power measured at the output of the POF sensor over time, during a time period of 30 days.

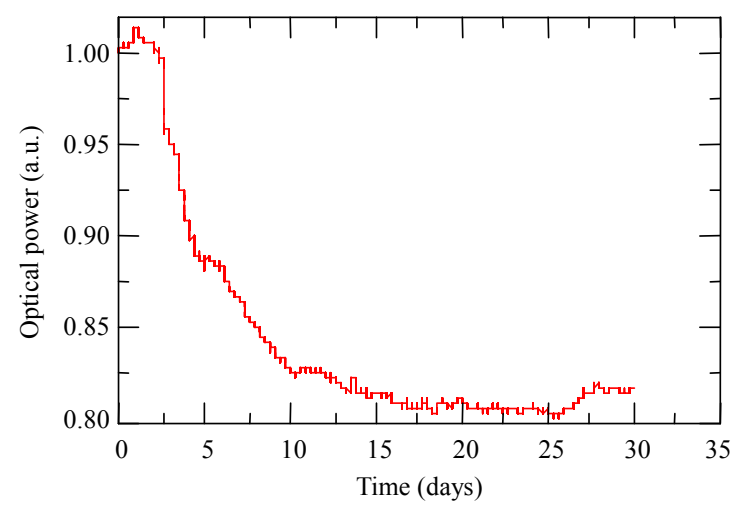

Fig. 7 Optical power evolution over time during the curing process.

Through the optical monitoring process, it was possible to measure different response stages during the curing process, due to a decrease in the water content in the concrete mixture. Associated with a decrease in the optical power was the light scattering, enhanced by the grooves in the POF. Therefore, the potential application of the optical POF sensor to the curing process monitoring optimization was demonstrated.

\section{Conclusions}

Optical fiber sensors were used for structural health monitoring. We proposed, implemented and tested monitoring schemes using static and dynamic sensors for the monitoring of different types of structural systems. The results illustrated the feasibility of using such technology with similar performance when compared with electronic sensors, however with advantages, as mentioned in the 
introduction.

\section{Acknowledgment}

Paulo Antunes acknowledges the financial support from Fundação para a Ciência e Tecnologia (FCT) through the Postdoctoral fellowship SFRH/BPD/76735/2011. The authors also thank for the support of University of Aveiro, Vodafone Portugal, Comunicações Pessoais, S. A. and Telcabo, Telecomunicações e Electricidade, Lda.

Open Access This article is distributed under the terms of the Creative Commons Attribution License which permits any use, distribution, and reproduction in any medium, provided the original author(s) and source are credited.

\section{References}

[1] P. Antunes, H. Lima, N. Alberto, L. Bilro, P. Pinto, A. Costa, et al., "Optical sensors based on fiber Bragg gratings for structural health monitoring," in New Developments in Sensing Technology for Structural Health Monitoring. S. C. Mukhopadhyay Ed. Heidelberg: Springer-Verlag, 2011, pp. 253-295.

[2] P. Antunes, H. Lima, J. Monteiro, and P. S. André, "Elastic constant measurement for standard and photosensitive single mode optical fibers," Microwave and Optical Technology Letters, vol. 50, no. 9, pp. 2467-2469, 2008.

[3] P. C. Chang, A. Flatau, and S. C. Liu, "Review paper: health monitoring of civil infrastructure," Structural Health Monitoring, vol. 2, no. 3, pp. 257-267, 2003.

[4]A. D. Grosso, K. Bergmeister, D. Inaudi, and U. Santa, "Monitoring of bridges and concrete structures with fiber optic sensors in Europe," in International Association for Bridge and Structural Engineering (IABSE) Conference, Seoul, Korea, August, pp. 15-22, 2001.

[5] J. M. Ko and Y. Q. Ni, "Technology developments in structural health monitoring of large-scale bridges," Engineering Structures, vol. 27, no. 12, pp. 1715-1725, 2005.

[6] C. Williamson and L. Fixter, "State of the art review: structural health monitoring," QinetiQ,
QinetiQ/S\&DU/T\&P/E\&M/TR0601122, 2006.

[7] D. Adams, Health monitoring of structural materials and components: methods with applications. Chichester: John Wiley \& Sons Inc., 2007.

[8] H. Wenzel and P. Furtner, "SHM at the civil infrastructure: applications, recent progress and future demands," 2010 (available from: http://www.brimos.com/Brimos/HTML/FRAMESET/ Frameset_Downloads.htm).

[9] P. F. C. Antunes, H. F. T. Lima, N. J. Alberto, H. Rodrigues, P. M. F. Pinto, J. L. Pinto, et al., "Optical fiber accelerometer system for structural dynamic monitoring," IEEE Sensors Journal, vol. 9, no. 11, pp. 1347-1354, 2009.

[10] M. Majumder, T. K. Gangopadhyay, A. K. Chakraborty, K. Dasgupta, and D. K. Bhattacharya, "Fiber Bragg gratings in structural health monitoring-present status and applications," Sensors and Actuators A: Physical, vol. 147, no. 1, pp. 150-164, 2008.

[11] A. Othonos and K. Kalli, Fiber Bragg Gratings: fundamentals and applications in telecommunications and sensing. Boston: Artech House, 1999.

[12] P. Antunes, F. Domingues, M. Granada, and P. André, "Mechanical properties of optical fibers," in Selected Topics on Optical Fiber Technology. M. Yasin, S. Harun, and H. Arof Ed. Rijeka, Croatia: InTech, 2011 (available from: http://www.intechopen.com/books/selected-topics-o n-optical-fiber-technology/mechanical-properties-ofoptical-fibers).

[13] K. S. C. Kuang, S. T. Quek, C. G. Koh, W. J. Cantwell, and P. J. Scully, "Plastic optical fiber sensors for structural health monitoring: a review of recent progress," Journal of Sensors, vol. 2009, pp. 312053-1-312053-13, 2009.

[14]A. Vallan, M. L. Casalicchio, A. Penna, and G. Perrone, "An intensity based fiber accelerometer," in 2012 IEEE International Instrumentation and Measurement Technology Conference (I2MTC), Graz, Austria, May 13-16, pp. 1078-1082, 2012.

[15] P. Antunes, H. Lima, H. Varum, and P. André, "Optical fiber sensors for static and dynamic health monitoring of civil engineering infrastructures: Abode wall case study," Measurement, vol. 45, no. 7, pp. 1695-1705, 2012.

[16] A. Mita and I. Yokoi, "Fiber Bragg grating accelerometer for structural health monitoring," presented at Fifth International Conference on Motion and Vibration Control (MOVIC 2000), 
Sydney, Australia, Dec. 4-8, 2000.

[17] P. F. C. Antunes, C. A. Marques, H. Varum, and P. S. Andre, "Biaxial optical accelerometer and high-angle inclinometer with temperature and cross-axis insensitivity," IEEE Sensors Journal, vol. 12, no. 7, pp. 2399-2406, 2012.

[18] P. S. André, H. Varum, P. Antunes, L. Ferreira, and M. G. Sousa, "Monitoring of the concrete curing process using plastic optical fibers," Measurement, vol. 45, no. 3, pp. 556-560, 2012.

[19] H. C. Pereira, "Caracterização do comportamento estrutural de construções em adobe," Civil engineering, Universidade de Aveiro, Aveiro, Portugal, MSc, 2008.

[20] P. Antunes, H. Rodrigues, J. Melo, H. Varum, and P. André, "Dynamic monitoring of an elevated water reservoir with an optical biaxial accelerometer," presented at OSA Sensors 2012, Monterey, USA, Jun. 24-28, 2012.

[21] P. Antunes, R. Travanca, H. Varum, and P. André, "Dynamic monitoring and numerical modelling of communication towers with FBG based accelerometers," Journal of Constructional Steel Research, vol. 74, pp. 58-62, 2012. 